\title{
Modulation of Quorum-Regulatory sRNA on Various Physiological Functions and Virulence of Vibrio alginolyticus
}

\author{
Huan Liu ${ }^{1}$, Jinfang Yang ${ }^{2}$, Shanshan Zhang ${ }^{3}$, Wang Liư ${ }^{4}$ Xuefeng Chen ${ }^{5}$ \\ ${ }^{1,2,3,4,5}$ School of Food and Biological Engineering, Shaanxi University of Science \& Technology, Xi'an \\ Shaanxi, 710021, China
}

Key words: Qrr1, Vibrio alginolyticus, Physiological function, Virulence.

\begin{abstract}
Vibrio alginolyticus is one of the important fish pathogen in China, bringing about serious economic loss to aquaculture. sRNA (Small non-coding RNA, sRNA) modulate the target genes on the post-transcriptional level with the aid of the chaperon protein, Hfq and play essential roles in the regulation of various physiological functions, like virulence. In this paper, a quorum regulatory sRNA, Qrr1, was screened and cloned. Besides, An in-frame deletion was made in qrr1 by allelic exchange, using the suicide plasmid. Meanwhile, the plasmid, pMMB206 was utilized to carry the contact $q r r 1$ gene into the mutant strain $\Delta$ qrr1 to generate the complemented strain, $q r r 1^{+}$. The roles of this gene in different physiological functions of Vibrio alginolyticus were characterized. The results demonstrated that Qrr1 obviously promoted the growth of Vibrio alginolyticus during exponential and stationary stage. Moreover, the ability of mobility was almost absent, the biofilm formation and extracellular protease production were all impaired in the $\Delta$ qrr1 mutant strain, contrast to the wild-type strain, which means Qrr1 participates in the virulence regulation as an important regulator in Vibrio alginolyticus.
\end{abstract}

\section{Introduction}

Vibrio alginolyticus is a kind of important aquatic pathogenic bacteria, widespread around the world, can infect a variety of fish and shellfish, bacterial septicemia caused by its infection brings great economic loss for aquaculture industry.

Toxic factors of vibrio alginolyticus include extracellaluar proteases, bacterial biofilm, moveability and so on, it is mainly regulated and controlled by quorum-sensing system and sRNA molecular chaperone protein Hfq(Liu et al. 2011; Gu et al. 2016). Hfq is initially identified as endogenous host protein that necessary for bacteriophage to replicate in the escherichia coli. Deletion analysis of Hfq reveals that this protein participates in the physiological regulation like growth of escherichia coli, cell division, response of the osmotic pressure(Tsui et al. 1994). Later, Hfq is founded in many bacteria, and it display regulation and control on the physiological function of bacteria(Chao and Vogel 2010). Hfq displays its regulation and control as a kind of uncoded sRNA chaperone proteins. sRNA is a kind of uncoded RNA with regulation and control functions in the bacteria, with size of 60 300 basic groups. sRNA can be rapid synthesis under the cooperated function of Hfq chaperone proteins, combine with different target mRNA through complementary base pairing, affecting its stability and translation activity to realize the rapid regulation and control on genetic expression. Hfq forms a six dimer structure of donut shape, with two independent bonding sites surfaces, one is in the far end and used for the combination of Poly (A tail), the other one is in the close end and is used for the combination of AU enrichment region. Therefore, Hfq provides RNA molecule with moored platform to promote the interaction of uncoded sRNA and target mRNA molecule (Mikulecky et al. 2004; Schu et al. 2015). Currently, more and more researches founded that Hfq and sRNAs has an essential effect on various physiological functions of many bacteria, such 
as growth features, environmental stress and virulence, when the Hfq is damaged, vibrio cholera, haemophilus bleeding and yersinia enterocolitica will be significantly attenuated(Lenz et al. 2004; Hempei et al. 2013; Kakoschke et al. 2014; Feliciano et al. 2016).

This research filtrates quorum sensing regulation sRNA molecule -- Qrrl in the splendidus vibrio genome through bioinformatics method, then to gain nullmutant for biological characteristics research through unmarked box method to gain qrrl gene from the genome.

\section{Materials and methods}

\section{Bacterial strains and growth conditions}

Bacterial strains and plasmids used in this study are listed in Table 1. Growth in Luria-Bertani (LB) broth or on $\mathrm{LB}$ plates containing $3 \%(\mathrm{w} / \mathrm{v}) \mathrm{NaCl}$ at $30^{\circ} \mathrm{C}$ was used for Vibrio alginolyticus culture in the whole study. Escherichia coli strains used for plasmid DNA replication and conjugation were grown in LB broth or medium containing $1 \%(\mathrm{w} / \mathrm{v}) \mathrm{NaCl}$ supplemented with appropriate antibiotics at $37^{\circ} \mathrm{C}$. And the antibiotics were used at the following concentrations: $100 \mu \mathrm{g} / \mathrm{ml}$ ampicillin (Amp) and $7 \mu \mathrm{g} / \mathrm{ml}$ chloramphenicol (Cm) for V. alginolyticus while $100 \mu \mathrm{g} / \mathrm{ml}$ Amp and $25 \mu \mathrm{g} / \mathrm{ml} \mathrm{Cm}$ for $E$. Coli.

Table 1 Strains and plasmids used in this study

\begin{tabular}{ccc}
\hline $\begin{array}{c}\text { Strains or } \\
\text { plasmids }\end{array}$ & Characteristics & Reference or source \\
\hline $\begin{array}{c}\text { Vibrio } \\
\text { alginolyticus }\end{array}$ & \\
EPGS & Pathogenic isolate from aquaculture farm. Ampr & Lab collected \\
asp- & EPGS carrying the null mutation in asp gene & Lab collected \\
$\Delta$ luxR & EPGS carrying in-frame deletion in luxR gene & Lab collected \\
$\Delta$ aphA & EPGS carrying in-frame deletion in aphA gene & Lab collected \\
$\Delta$ qrr1 & EPGS carrying in-frame deletion in qrr1 gene & This study \\
qrr1+ & EPGS, $\Delta$ hfq complemented with intact hfq gene & This study \\
Plasmids & Cmr, pDM4 derivative containing the truncated qrr1 & This study \\
pDM4- $\Delta$ qrr1 & from bp 7-121 & This study \\
pMMB-qrr1 & Cmr, pMMB206 derivative containing the intact qrr & \\
\hline
\end{tabular}

\section{DNA manipulation}

All DNA manipulations were carried out as previously described. Enzymatic reactions and plasmid purifications were performed according to the manufacturer's instruction (TaKaRa, Dalian, China), and DNA sequencing and primer synthesis were finished in Generay Co. (Shanghai, China).

Clone and analysis of qrrl gene

According to the published vibrio alginolyticus 12G01 genome, vibrio cholera and qrrl gene order in the vibrio harveyi, make compare analysis by using bioinformatics method, choose quorum sensing regulation sRNA and Qrrl in the vibrio alginolyticus to design specific primers to clone qrrl gene and use Mfold to make secondary structure prediction analysis.

The construction of $\Delta$ qrrl mutant strain

Set vibrio alginolyticus EPGS genome as template, respectively amplify primers, qrr1upF and qrr1upR, qrr1doF and qrr1doR to get upstream and downstream homologous segments -- qrr1up and qrr1do. After recycling, PCR products connect upstream and downstream homologous segments qrr1up and qrr1do through overlap PCR by using qrr1upF and qrr1doR as primers to get $\Delta$ qrr1 fragment. Use suicide plasmid pDM4 to combine vibrio alginolyticus EPGS to get PCR and correctly verified mutant strain $\Delta$ qrr1 through two rounds of homologous recombination.

The construction of complementary strain qrr1+ 
Set EPGS genome as template, use primers qrr1comF and qrr1comR to carry out PCR reaction, enlarge to get DNA fragment with complete qrr1 gene ORF region, use complementary plasmid pMMB206 to integrate it into the recipient bacterium $\Delta$ qrr1 to get complementation strain $q r r 1^{+}$.

The measurement of growth curve

Inoculate bacterial strain that keeping in the $-80^{\circ} \mathrm{C}$ glycerin tube into LBS fluid medium, $200 \mathrm{r} / \mathrm{min}$ resuscitaion culture overnight in the $30^{\circ} \mathrm{C}$ table concentrator. Use fresh LBS fluid medium to dilute $\mathrm{OD}_{600} \mathrm{~nm}$ to 1.0 , inoculate it into $50 \mathrm{ml}$ LBS fluid medium according to $1 \%(\mathrm{v} / \mathrm{v})$, culture with 200 $\mathrm{r} / \mathrm{min}$ in the $30^{\circ} \mathrm{C}$ table concentrator, sampling every $1 \mathrm{~h}$ and measure the $\mathrm{OD}_{600} \mathrm{~nm}$ to draw the growth curve of each bacterial strain.

Detection of biofilm

Use fresh LBS fluid medium to dilute the overnight cultured bacterial strain to same $\mathrm{OD}_{600 \mathrm{~nm}}$ value. Then inoculate each bacterial strain to sterile tubes with $10 \mathrm{ml}$ LBS fluid medium, stationary culture for $48 \mathrm{~h}$ under $30^{\circ} \mathrm{C}$. Each tube added with $2 \%$ (w/v) crystal violet solution, dyeing for 5 minutes, pour out the culture and wash with tap water, each tube added with 33\% (v/v) glacial acetic acid solution, detect its light absorption value at $570 \mathrm{~nm}$.

Dynamic analysis

Respectively dot same amount activated vibrio alginolyticus EPGS, $\triangle$ qrr1 mutant strain and $\operatorname{qrr}^{+}$complementary strains on the LBS slab with $0.3 \%(\mathrm{w} / \mathrm{v})$ (soft slab) and $1.5 \%(\mathrm{w} / \mathrm{v})$ (hard slab) agar powder, culture under $30^{\circ} \mathrm{C}$ until bacterial colony grows to reasonable size, take out for photo and measure the diameter.

Activity analysis of extracellaluar proteases

Qualitative determination use method of skin milk slab, quantitative analysis is to measure the amount of soluble sky blue dye released by the enzymolysis of Hide powder azure, HPA. Measuring method refers to Liu et al. 2011.

\section{Results}

\section{Qrr1 order analysis and the construction of $\Delta$ qrr1 mutant strain}

Set vibrio alginolyticus EPGS genome as template to enlarge qrr1 order to 121 bp and keep high homology with vibrio harveyi, vibrio parahaemolyticus, qrr1 order in the vibrio cholera. Use Mfold online analytical tools to make secondary structure prediction for the transcription RNA, we found that Qrr1 has typical stem loop structure, its 3'normally has U tail, and there is AU-rich region in the assumed Hfq binding site in the stem loop structure.

Further to get the 7-121, 115bp mutant strain $\Delta$ qrr1 of deficiency qrr1. PCR verification and order measurement result showed that 7-121 basic groups totally 115bp internal nucleotide of mutant strain $\Delta$ qrr1 was missing.

\section{The construction of qrr1+ complementary strains}

Connect complementary fragments into pMMB206 plasmid and combine into $\Delta$ qrr1 mutant strain on the basis of mutant strain. Use primers qrr1comF and qrr1comR to make PCR verification of bacterial strain. The result showed that the $q r r 1^{+}$complementary strains can enlarge to get two fragments differ $115 \mathrm{bp}$, that is the frame missing fragment in the $\Delta$ qrr1 mutant strain and the complete complementary fragment of pMMB206 plasmid that can start subdomain, which proves that covering plasmid pMMB-qrr1 with target fragment is successfully transited to the $\Delta$ qrr1 mutant strain.

\section{Influence on the growth of vibrio alginolyticus}

In order to investigate the function of Qrrl on the growth of vibrio alginolyticus, we respectively measure the growth curve of vibrio alginolyticus wild type strain and complementary strain. The result showed that all of them get into logarithmic phase after being inoculated into the fresh nutrient 
medium; wild type strain grows fast in the logarithmic phase, in the stable phase, thallus intensity reaches $\mathrm{OD}_{600} \mathrm{~nm}=6.0$. During the logarithmic phase, compared with wild type strain, the growth of mutant strain and complementary strain declines obviously; after getting into the stable phase, the growth of mutant strain reaches the growth of complementary phase, wild type strain keeps higher germ concentration. It is visible that the missing of Qrrl gene has influence on the whole growth period of vibrio alginolyticus, has biggest influence on the logarithmic phase.

\section{The regulation and control of Qrrl on vibrio alginolyticus moveability}

Moveability detection result showed that wild type strain has strong moveability on hard slab and soft slab, on the hard slab, it creep diffuses to surrounding from the sampling dot, it almost covers the whole soft slab. While the moveability of $\Delta$ qrr1 mutant strain almost lost. It is visible that the positive regulation function of Hgq on vibrio alginolyticus is mainly realized from the function of Qrrl.

\section{Influence of Qrr1 on the biofilm formation of vbrio alginolyticus}

Through detection on the biofilm of different bacterial strain we found that vbrio alginolyticus wild type can form visible and thick biofilm on the surface of test tube wall and LBS fluid nutrient medium. Under the same culture condition, the biofilm of $\Delta$ qrr1 mutant strain is reduced, while though the biofilm formation of $q r r 1^{+}$complementary strain did not reach that of the wild type, but has obvious increase. Through crystal violet staining analysis we found that the biofilm formation of $\Delta$ qrr1 mutant strain is reduced by 50\%, compared with the wild type. It is visible that Qrr1 has positive regulation function on the biofilm formation of vbrio alginolyticus.

\section{Influence of Qrr1 on the extracellaluar proteases formation of vbrio alginolyticus}

Wild type bacterial strain can form obvious transparent zone around the bacterial colony on the skim milk slab, and the diameter and transparency of the transparent zone are biggest. While the diameter and transparency of the transparent zone of the $\Delta$ qrr1 mutant strain are decreased; when there is complete gene open reading order complementary to the mutant strain, its extracellaluar proteases activity will be improved on a certain extent. The HPA cromogenic reaction results conform with milk slab, the yield of extracellaluar proteases of the wild type is highest, when Qrr1 is damaged, its acellaluar proteases production is lowest, and the production of complementary strain is close to the wild type. It is visible that Qrr1 has some promotion on the production of extracellaluar proteases of vbrio alginolyticus.

\section{Discussion}

The main virulence factorsof vbrio alginolyticus are racellaluar proteases, biofilm and moveability. Former researches found that there is sRNA chaperone protein - Hfq in the vbrio alginolyticus, this protein has important regulations on the environmental stress ability, moveability, biofilm, extracellular protease activity, survival in the host and virulence of the vbrio alginolyticus (Liu et al. 2011). As an important transcription regulatory factor, Hfq mainly assists the interaction of different sRNA molecule and target mRNA moluce. In the escherichia coli, Hfq displays regulation on nutrition metabolism through two sRNA molecules, SgrS and CsrA (Papenfort et al. 2013; Wang et al. 2005); displays oxidative stress through the transition of fhIA and rpoS gene(Updegrove and Wartell. 2011). In the vibrio cholera, Hfq mainly realizes its regulation on virulence through the influence of Orr molecule on the quorum sensing key components LuxR and AphA (Shao and Bassler. 2012).

This paper screened quorum sensing regulation sRNA molecule-Qrr1 from the 12G01 genome of vbrio alginolyticus by using bioinformatics method. Establish a mutant strain missing 7-121 basic group, through comparing its different physiological property with wild type bacterial strain and complementary strain we found that Qrr1 has some promotion on the synthesis of extracellaluar proteases of vbrio alginolyticus, while former researches found that when Hfq is missing, its extracellaluar proteases production is obviously improved, it is visible that in the vbrio alginolyticus, 
Hfq mainly affect extracellaluar proteases trough one or many sRNA but not Qrr1. When Qrr1 is missing, the moveability of vbrio alginolyticus totally lost, which is same with the Hfq missing strain, which showed that the regulation of Hfq on the moveability of vbrio alginolyticus is mainly realized by Qrr1. Besides, when the Qrr1 is damaged during transition, only the cell concentration of vbrio alginolyticus in the logarithmic phase and stable phase is obviously lower than wild type, while when the Hfq is damaged, only the cell concentration of vbrio alginolyticus in the logarithmic phase is decreased a little by comparing with the wild type, when in the stable phase, it reaches the level of the wild type, which showed that Hfq displays regulation on the growth and metabolism of vbrio alginolyticus through Qrr1 and other unknown sRNA molecule.

Through research in this paper, we identified the sRNA molecule - Qrr1, which is related with the growth and virulence of vbrio alginolyticus, provided the regulation of Hfq on the virulence and other physiological functions with reference. Meanwhile, research results also showed that the complicity of Hfq's virulence regulation system on vbrio alginolyticus, there are still a large number of unknown sRNA molecules which take part in the different physiological function of vbrio alginolyticus need frther identification.

\section{Acknowledgements}

This study was supported by grants from National Natural Science Foundation of China (No. 31301059), Shaangxi University of Science and Technology (No. BJ12-24) and Science and Technology Agency of Shaanxi Province (No. 2013JQ3011).

\section{References}

[1] Chao Y, Vogel J. The role of Hfq in bacterial pathogens, Curr Opin Microbiol, 2010, 13(1): 24-33.

[2] Feliciano JR, Grilo AM, Guerreiro SI, Sousa Leitão JH. Hfq: a multifaceted RNA chaperon involved in virulence, Future Microbiol. 2016, 11(1): 137-151.

[3] Gu D, Liu H, Yang Z, Zhang Y, Wang Q. Chromatin innunoprecipitation sequencing technology reveals global regulatory roles of low-cell-density quorum-sensing regulator AphA in the pathogen Vibrio alginolyticus, J Bacteriol, 2016, 198(21): 2985-2999.

[4] Hempei RJ, Morton DJ, Seale TW, Whitby PW, Stull TL, The role of RNA chaperon Hfq in Haemophilus influenza pathogenesis, BMC Microbiol, 2013, 13: 134.

[5] Kakoschke T, Kakoschke S, Magistro G, Schubert S, Borath M, Heesemann J, Rossier O. The RNA chaperon Hfq impacts growth, metabolism and production of virulence factors in Yersinia enterocolitica, PLoS One, 2014, 9(1): e86113.

[6] Lenz DH, Mok KC, Lilley BN, Kulkarni RV, Wingreen NS, Bassler BL, The small RNA chaperon Hfq and multiple small RNAs conrol quorum sensing in Vibrio harveyi and Vibrio cholerae, Cell, 2004, 118(1): 69-82.

[7] Liu H, Wang Q, Liu Q, Cao X, Shi C, Zhang Y. Roles of Hfq in the stress adaptation and virulence in fish pathogen Vibrio alginolyticus and its potential application as a target for live attenuated vaccine, Appl Microbiol Biotechnol, 2011, 91(2): 353-364.

[8] Mikulecky PJ, Kaw MK, Brescia CC, Takach JC, Sledjeski DD, Feig AL. Escherichia coli Hfq has distinct interaction surfaces for DsrA, rpoS and poly (A) RNAs, Nat Struct Mol Biol, 2004, 11(12): 1206-1214.

[9] Papenfort K, Sun Y, Miyakoshi M, Vanderpool CK, Vogel J. Regulation of glucose homeostasis by small RNA mediated activation of sugar phosphatase mRNA, Cell, 2013, 153(2): 426-437. 
[10]Schu DJ, Zhang A, Gottesman S, Storz G. Alternative Hfq-sRNA interaction modes dictate alternative mRNA recognition, EMBO J, 2015, 34(20): 2557-2573.

[11] Shao Yi, Bassler BL. Quorum-sensing non-coding small RNAs use unique pairing regions to differentially control mRNA targets, Mol Microbiol, 2012, 83(3):599-611.

[12]Tsui HCT, Leung HCE, Winkler ME. Characterization of broadly pleiotropic phenotypes caused by an hfq insertion mutation in Escherichia coli K-12, Mol Microbiol, 1994, 13(1): 35-49.

[13] Updegrove TB, Wartell RM. The influence of Escherichia coli Hfq mutation on RNA binding and sRNA mRNA duplex formation in rpoS ribo regulation, BBA-Gene Regul Mec, 2011, 1809(10):532-540.

[14]Wang X, Dubey AK, Suzuki K, Baker CS, Babitzke P, Romeo T. CsrA post-transcriptionally represses pgaABCD, responsible for synthesis of a biofilm polysaccharide adhesion of Escherichia coli, Mol Microbiol, 2005, 56(6):1648-1663. 